\title{
Quasi-particle model of strongly interacting matter
}

\author{
Marcus Bluhm $\dagger$, Burkhard Kämpfer $\dagger$ and Gerhard Soff $\ddagger$ \\ $\dagger$ Forschungszentrum Rossendorf, PF 510119, 01314 Dresden, Germany \\ $\ddagger$ Institut für Theoretische Physik, TU Dresden, 01062 Dresden, Germany \\ E-mail: M.Bluhm@fz-rossendorf.de
}

\begin{abstract}
The successful quasi-particle model is compared with recent lattice data of the coefficients in the Taylor series expansion of the excess pressure at finite temperature and baryon density. A chain of approximations, starting from QCD to arrive at the model expressions for the entropy density, is presented.
\end{abstract}

\section{Introduction}

The equation of state of strongly interacting matter at finite temperature $T$ and small chemical potential $\mu$ has become accessible fairly recently through first principle lattice QCD calculations 11, 2, 3, 4. Apart from solving QCD on the lattice, there exist analytical approaches such as resummed HTL scheme, $\Phi$ functional approach etc. (cf. [5] for a recent survey). Ab initio approaches [6, 7] are restricted in describing lattice data on temperatures $T \gtrsim 2.5 T_{\mathrm{c}}$, where $T_{\mathrm{c}}$ is the transition temperature of deconfinement and chiral symmetry restoration. In contrast, phenomenological models with adjustable parameters [8, 9, 10] cover the region $T \gtrsim T_{\mathrm{c}}$. Here, we present new developments of our quasi-particle model (QPM) [8, 9].

In section 2 the QPM is reviewed. In section 3 the model is confronted with recent lattice QCD data. Motivating our model, section 4 briefly illustrates a chain of approximations within a $\Phi$ funtional approach. The results are summarized in section 5 ,

\section{Quasi-particle model}

The model is based on the idea that the quark-gluon fluid can be expressed in terms of quasi-particles. The pressure of $N_{q}$ light (q), strange (s) quarks and gluons (g) reads

$p(T, \mu)=\sum_{i=q, s, g} p_{i}(T, \mu)-B(T, \mu)$.

$p_{i}$ are thermodynamic standard expressions in which $T$ and $\mu$ dependent self-energies $\Pi_{i}$ enter. $B(T, \mu)$, together with the stationarity condition $\delta p / \delta m_{i}^{2}=0$ [11], ensures 
thermodynamic self-consistency (cf. [9, 12] for details). Thus, the entropy density $s=\partial p / \partial T=\sum_{i=q, s, g} s_{i}$ explicitly reads

$s_{i}=\frac{d_{i}}{2 \pi^{2} T} \int_{0}^{\infty} \mathrm{d} k k^{2}\left\{\frac{\left(\frac{4}{3} k^{2}+m_{i}^{2}\right)}{\omega_{i}(k)}\left[f_{i}^{+}(k)+f_{i}^{-}(k)\right]-\mu\left[f_{i}^{+}(k)-f_{i}^{-}(k)\right]\right\}$

with statistical distribution functions $f_{i}^{ \pm}(k)=\left(\exp \left[\left(\omega_{i}(k) \mp \mu_{i}\right) / T\right]+S_{i}\right)^{-1}, S_{q}=S_{s}=1$, $S_{g}=-1, \mu_{q}=\mu, \mu_{s}=\mu_{g}=0$ and degeneracies $d_{q}=6 N_{q}, d_{s}=6$ and $d_{g}=8$.

In the thermodynamically relevant region of momenta $k \sim T$, $\mu$, the quasi-particle dispersion relations are approximated by the asymptotic mass shell expressions near the light cone $\omega_{i}^{2}(k)=k^{2}+m_{i}^{2}$ with $m_{i}^{2}=\Pi_{i}(k ; T, \mu)+\left(x_{i} T\right)^{2}$. The self-energies $\Pi_{i}$ of the quasi-particle excitations are approximated by their 1-loop expressions at hard momenta 9] neglecting imaginary parts, and $x_{i} T$ represent the quark masses used on the lattice [1]. Replacing the running coupling in the self-energies by an effective coupling, $G^{2}(T, \mu)$, non-perturbative effects are thought to be taken into account [12. Imposing thermodynamic consistency onto $p$, a flow equation for $G^{2}(T, \mu)$ follows [9]

$a_{\mu} \frac{\partial G^{2}}{\partial \mu}+a_{T} \frac{\partial G^{2}}{\partial T}=b$

which can be solved as Cauchy problem by knowing $G^{2}$ on an arbitrary curve $T(\mu)$. One convenient choice is parametrizing $G^{2}(T(\mu=0))$ appropriately (cf. [13]) such that $p$ and $s$ can be computed at non-vanishing $\mu$ from (12).

\section{Expansion coefficients}

Apart from (11), the pressure can be decomposed into a Taylor series

$\frac{p(T, \mu)}{T^{4}}=\frac{p(T, \mu=0)}{T^{4}}+\frac{\Delta p(T, \mu)}{T^{4}}=\sum_{n=0}^{\infty} c_{2 n}(T)\left(\frac{\mu}{T}\right)^{2 n}$

with $c_{0}(T)=p(T, \mu=0) / T^{4}$ and vanishing $c_{k}$ for odd $k$. The expansion coefficients $c_{k}$ have been subject of recent lattice evaluations [1] by computing derivatives of the thermodynamic potential. They follow from (11) as $c_{k}(T)=\partial^{k} p /\left.\partial \mu^{k}\right|_{\mu=0} T^{k-4} / k$ ! in our model and read

$c_{2}=\frac{3 N_{q}}{\pi^{2} T^{3}} \int_{0}^{\infty} \mathrm{d} k k^{2} \frac{\mathrm{e}^{\omega_{q} / T}}{\left(\mathrm{e}^{\omega_{q} / T}+1\right)^{2}}$,

$c_{4}=\frac{N_{q}}{4 \pi^{2} T^{3}} \int_{0}^{\infty} \mathrm{d} k k^{2} \frac{\mathrm{e}^{\omega_{q} / T}}{\left(\mathrm{e}^{\omega_{q} / T}+1\right)^{4}}\left\{\mathrm{e}^{2 \omega_{q} / T}-4 \mathrm{e}^{\omega_{q} / T}+1-\frac{T}{\omega_{q}}\left[\mathrm{e}^{2 \omega_{q} / T}-1\right] A_{2}\right\}$,

where $A_{2}=\left.\left(G^{2} / \pi^{2}+3 x_{q} \sqrt{G^{2} /\left(6 \pi^{4}\right)}+\left[\frac{3}{2} x_{q} T^{2} / \sqrt{6 G^{2}}+\frac{1}{2} T^{2}\right] \partial^{2} G^{2} / \partial \mu^{2}\right)\right|_{\mu=0}, \omega_{q}$ is taken at $\mu=0$ and $\partial^{2} G^{2} / \partial \mu^{2}$ follows from differentiating (3).

In the left panel of Figure 1, the QPM results of $c_{2,4}$ calculated from (156) are compared with lattice QCD results [1] for the two-flavour case, i.e. $N_{q}=2$. Using $x_{q}=0.4$ and $x_{g}=0$ as in [1] and setting $T_{\mathrm{c}}(\mu=0) \equiv T_{0}=170 \mathrm{MeV}, G^{2}(T(\mu=0))$ is adjusted to describe $c_{2}(T)$. Since $c_{4}$ in (6) only depends on $G^{2}$ and its derivatives at 
$\mu=0$, no further assumptions enter into the evaluation once the parametrization is fixed. A fairly good agreement is found for $c_{4}(T)$. Note, in particular, that the pronounced peak structure of $c_{4}$ at $T_{0}$ solely originates from the term including $\partial^{2} G^{2} /\left.\partial \mu^{2}\right|_{\mu=0}$.
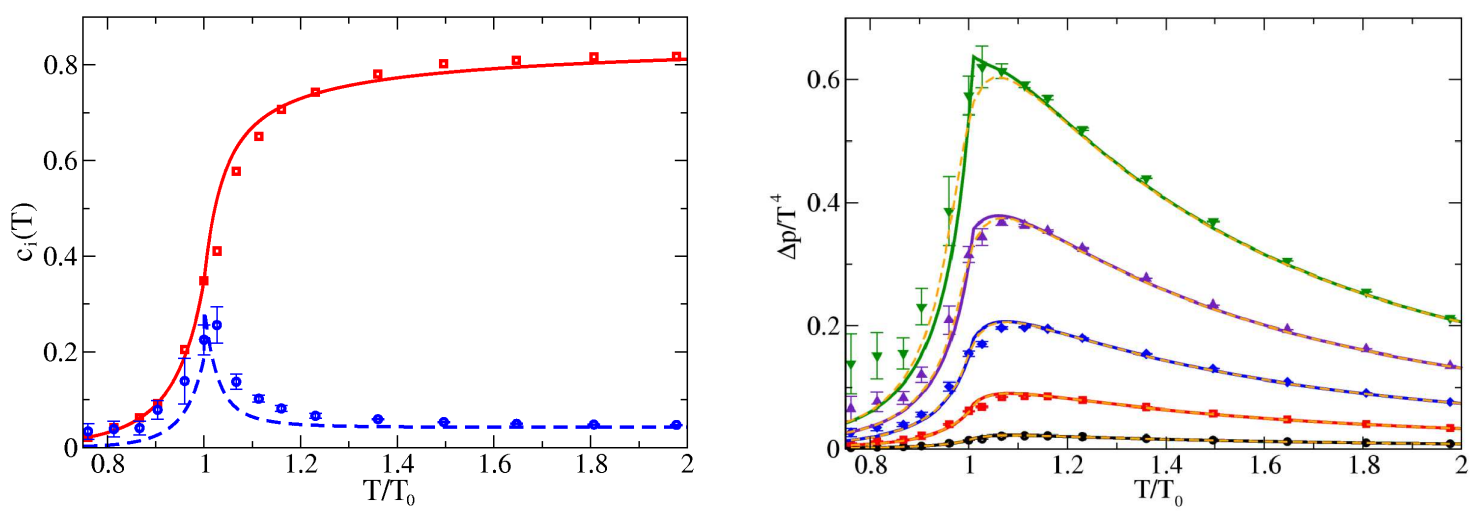

Figure 1. Left: Expansion coefficients $c_{2}(T)$ (squares) and $c_{4}(T)$ (circles). Data from 11. Full and dashed curves represent, respectively, corresponding QPM results (516). Right: Scaled excess pressure $\Delta p$ from [1] (symbols) and QPM results (full lines) from truncating (44) at order $(\mu / T)^{4}$ for different $\mu / T_{0}=0.2,0.4,0.6,0.8,1.0$ (lower to upper curve). Dashed lines depict corresponding full QPM results.

In [1, the excess pressure $\Delta p$ has been calculated as the truncation of the expansion (4) including the order $(\mu / T)^{4}$. The right panel of Figure 1 exhibits the comparison of $\Delta p$ calculated by employing only $c_{2,4}$ in (41) (full lines) with the lattice data for different $\mu / T_{0}$. An impressively good agreement with the data is observed for small values of $\mu$ in which case $c_{4}$ is of less importance. Similarly, $\Delta p$ can be evaluated as infinite series from the QPM by combining (11) and (4) (dashed lines). These full results differ noticeably from the truncated results only for $T \approx T_{0}$. It should be noted that the model is successfully applied to describing the equation of state with strange quarks [14.

\section{Contact with QCD}

Motivating the strong assumptions made in formulating the QPM expressions (12), a chain of reasonable approximations starting from QCD would be of desire. The thermodynamic potential $\Omega=-p V$ in ghost free gauge reads [6]

$\Omega[D, S]=T\left\{\frac{1}{2} \operatorname{Tr}\left[\ln D^{-1}-\Pi D\right]-\operatorname{Tr}\left[\ln S^{-1}-\Sigma S\right]\right\}+T \Phi[D, S]$

with dressed propagators $D$ and $S$ of bosons and fermions and corresponding exact self-energies $\Pi$ and $\Sigma$ from Dyson's equations. The functional $\Phi$ is given by the sum over all 2 particle irreducible skeleton diagrams and $\Pi, \Sigma$ follow from truncating dressed 
propagator lines in these diagrams [6]. The sum over Matsubara frequencies in the trace $\mathrm{Tr}$ is transformed into an appropriate contour integral in the complex energy plane. Computing the entropy density $s=-\partial(\Omega / V) / \partial T$, an ultra-violet finite expression

$$
\begin{aligned}
s= & -\operatorname{tr} \int \frac{d^{4} k}{(2 \pi)^{4}} \frac{\partial n(\omega)}{\partial T}\left[\operatorname{Im} \ln D^{-1}-\operatorname{Im} \Pi \operatorname{Re} D\right] \\
& -2 \operatorname{tr} \int \frac{d^{4} k}{(2 \pi)^{4}} \frac{\partial f(\omega)}{\partial T}\left[\operatorname{Im} \ln S^{-1}-\operatorname{Im} \Sigma \operatorname{Re} S\right]+s^{\prime}
\end{aligned}
$$

is derived. After truncating $\Phi$ at 2 loop order, $s^{\prime}=0$ is found. Lost gauge invariance gets restored by employing hard thermal loop expressions for $\Pi$ and $\Sigma$ which show the correct limiting behaviour for $k \sim T, \mu$. Performing the remaining trace tr over discrete indices in (8), quantum numbers of quarks and gluons are recovered. Furthermore, the exponentially suppressed longitudinal gluon modes and the plasmino branch can be neglected. In addition, neglecting imaginary parts in $\Pi$ and $\Sigma$ as well as Landau damping and approximating self-energies and dispersion relations suitably, expression (2) for the entropy density $s$ is recovered.

\section{Conclusion}

The quasi-particle model has been reviewed and successfully compared with recent lattice data of the expansion coefficients $c_{2,4}$ and the excess pressure at finite temperature and chemical potential. Briefly, a chain of approximations within the $\Phi$ functional scheme starting from QCD has been summarized which leads to our model.

\section{Acknowledgments}

Inspiring discussions with A Peshier are gratefully acknowledged. The work is supported by BMBF, GSI and EU-I3HP.

\section{References}

[1] Allton C R et al 2003 Phys. Rev. D 68014507

[2] Csikor F et al 2004 J. High Energy Phys. JHEP05(2004)046; 2004 Prog. Theor. Phys. Suppl. 153 93-105

[3] Fodor Z and Katz S D 2002 Phys. Lett. B 534 87-92

[4] Fodor Z et al 2003 Phys. Lett. B 568 73-7

[5] Rischke D H 2004 Prog. Part. Nucl. Phys. 52 197-296

[6] Blaizot J P et al 1999 Phys. Lett. B 470 181-8

[7] Blaizot J P et al 2002 Nucl. Phys. A 698 404-7

[8] Peshier A et al 1994 Phys. Lett. B 337 235-9; 1996 Phys. Rev. D 54 2399-402

[9] Peshier A et al 2000 Phys. Rev. C 61045203

[10] Levai P and Heinz U 1998 Phys. Rev. C 57 1879-90 Schneider R A and Weise W 2001 Phys. Rev. C 64055201

Letessier J and Rafelski J 2003 Phys. Rev. C 67031902

Rebhan A and Romatschke P 2003 Phys. Rev. D 68025022

Thaler M A et al 2004 Phys. Rev. C 69035210 
[11] Gorenstein M I and Yang S N 1995 Phys. Rev. D 52 5206-12

[12] Peshier A et al 2002 Phys. Rev. D 66094003

[13] Bluhm M et al 2004 Preprint hep-ph/0402252 2004 Preprint hep-ph/0411106

[14] Szabo K K and Toth A I 2003 J. High Energy Phys. JHEP06(2003)008 